\title{
Homöopathie und Bergkrankheit: Selbsterfahrung in Nepal
}

\author{
Urs Steiner \\ Arzt für Allgemeinmedizin/Klassische Homöopathie, $\mathrm{CH}-I m m e n s e e$
}

$D^{\prime}$ ie Höhenkrankheit umfasst klinisch zwei Formen, das Höhenlungen- und das Höhenhirnödem, wobei das Höhenhirnödem bedeutend häufiger auftritt, manchmal auch assoziiert ist mit dem Lungenödem. Sie entsteht bei einem zu schnellen Anstieg, doch scheinen auch genetische Faktoren mitzuspielen. Der SauerstoffPartialdruck nimmt pro 1000 Höhenmeter um 10\% ab. Die Höhenkrankheit oder einzelne Symptome davon können bei schnellem Aufstieg (z.B. nach einer Autofahrt vom Meer direkt ins Hochgebirge) schon in geringen Höhen, z.B. auf 2500 m, auftreten. Ab Höhen über 2500 m sollte der Aufstieg anfänglich $700 \mathrm{~m}$ pro 24 Stunden nicht überschreiten, ab $4000 \mathrm{~m}$ dürfte die Grenze bei etwa $500 \mathrm{~m}$ und ab $5000 \mathrm{~m}$ bei etwa $300 \mathrm{~m}$ pro 24 Stunden liegen. Die vorliegende Arbeit berücksichtigt nur die cerebrale Form der Höhenkrankheit, deren progressive Symptome sind: Kopfschmerz klopfender, bohrender, reissender oder stechender Art, schneller Puls, Übelkeit, Erbrechen, Benommenheit, Schwindel, Gangataxie, Verwirrungszustände, Wahnvorstellungen. Die schulmedizinische Standardtherapie der Höhenkrankheit ist der Calcium-Antagonist Nifedipin (v.a. bei der pulmonalen Form), kombiniert im Extremfall auch bei der cerebralen Form mit Dexa-Methason und begleitet von einem schnellen Abstieg [1,2,3]. Homöopathisch kommen mehrere Arzneien in die engere Wahl, so z.B. nach Erfahrung des Autors Apis, Arnica, Belladonna, Glonoin, Lachesis [4,5,6].

Am 27. Oktober 2005 startete ich zusammen mit meinem Sherpa und

Die akute Bergkrankheit mit schweren Kopfschmerzen verläuft ziemlich uniform, verlangt aber homöopathisch eine strenge individuelle Therapie, wobei die Wahl aus einem halben Dutzend Arzneien getroffen werden muss. Eine Ausmittelung am Tisch mit Computer oder Buch-Repertorium dürfte in den seltensten Fällen möglich sein, was eine genaue Kenntnis der einzelnen Arzneien voraussetzt. Praktische Erfahrung konnte der Autor unter anderem bei Besteigungen des Kilimanjaro (5893 m) in Tanzania, des Jala Peak (5600 m) und des Island-Peak (6165 m) in Nepal sammeln. Letztere Tour ist Anlass aufzuzeigen, dass Kopfschmerz auch im Gebirge multifaktoriell sein kann, dass derselbe unbehandelt zum Höhenhirnödem führen kann und eine systematische Symptomenerhebung unabdingbar ist. Diese führt zur Wahl eines spezifischen Arzneimittels, das dem Bergsteiger erlaubt, den Aufstieg beschwerdefrei fortzusetzen.

Schlüsselwörter: Bergkrankheit, Kopfschmerzen, Homöopathie, Fallbeispiel

\section{Homeopathy and Mountain Sickness: A Personal Experience in Nepal}

High altitude sickness with severe headache is an acute condition with more or less uniform symptoms. To be cured it nevertheless needs an individualized choice of a homoeopathic medicine selected from around half a dozen possibles. Repertorisation under field conditions is usually not possible so that good acquaintance with the drugs in question is a precondition. The author describes his personal experience on a climbing tour to the Island Peak $(6165 \mathrm{~m})$ in Nepal. On other occasions such as trekking to the Kilimanjaro (5893 meters) in Tanzania and climbing to the Jala Peak (5600 meters) in Nepal he observed that headaches at high altitudes may have different causes, and some may lead to an edema of the brain if untreated. An exact evaluation of the symptoms is of great importance and leads to the choice of a specific homeopathic medicine, which allows the patient to continue the ascent without risk.

Keywords: Mountain sickness, severe headache, homeopathy, case report

den zwei Porters vom 4735 Meter hohen Chhukhung zur letzten Wasserstelle des Island Peak auf 5250 Meter mit dem Ziel, noch in der Nacht zum $6165 \mathrm{~m}$ hohen Gipfel aufzusteigen (Abb. 1). Ich war mir bewusst, dass die eingeplante Aufstiegsgeschwindigkeit von 1430 Metern in 24 Stunden weit über der physiologischen Limite lag, doch ich fühlte mich so gut, dass ich trotz meiner 62 Jahre keine Bedenken hatte. Mein Trainingszustand liess auch nichts zu wünschen übrig, jogge ich doch seit über 40 Jahren etwa dreimal wöchentlich, meistens am Berg, und absolviere noch zusätzlich zeitweilig ein leichtes Krafttraining. Wir errichteten unser Zelt, um uns einige Stunden Wärme und Schlaf zu gönnen. Es war bitter kalt, der Wind blies und an der Eiswand gegenüber war das Furcht einflössende Donnern niedergehender Lawinen zu vernehmen. In einem geschützten Winkel unseres Zeltes kochten wir uns Tee und Dal Bad, das typische Reis-LinsenGericht, Appetit hatte ich allerdings keinen, was bei anstrengenden Touren 


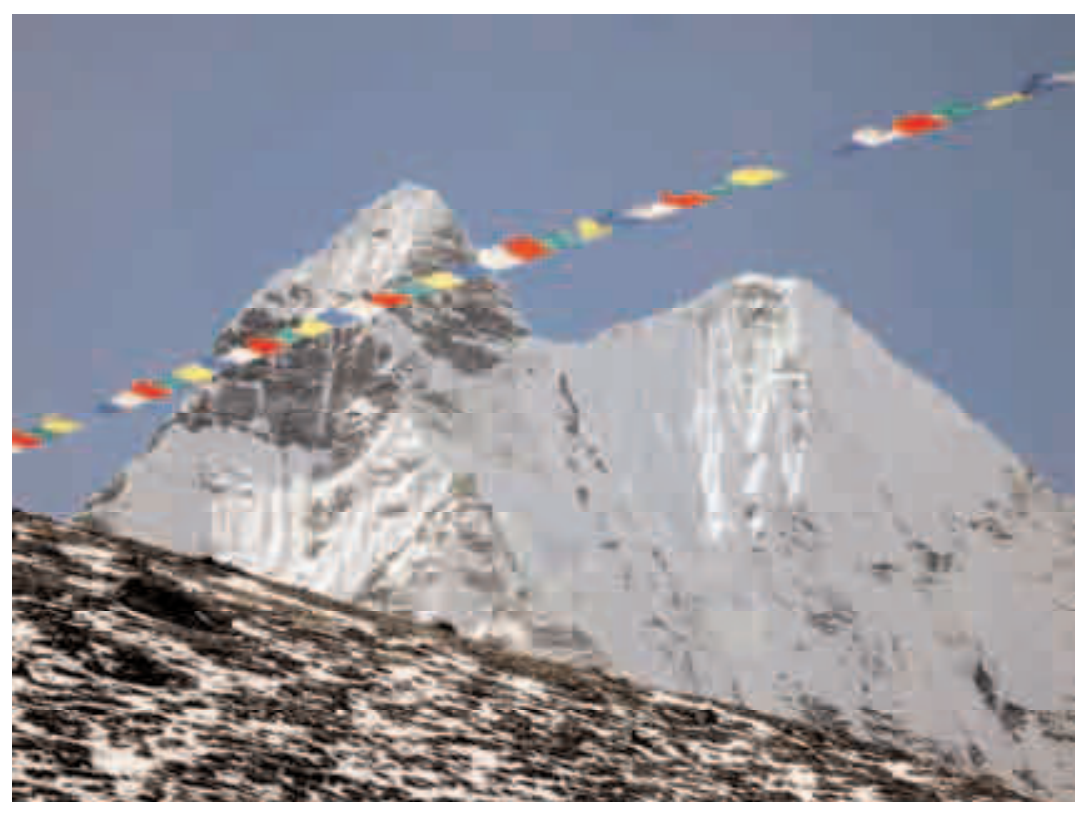

Abb. 1. Island-Peak, Nepal (6165 m).

für mich aber normal war. Wegen der Kälte und der Dunkelheit schlüpften wir schon um 18.30 Uhr in unsere wärmenden Daunen-Schlafsäcke. Um 02.15 Uhr sollten wir uns wieder erheben, wir versuchten deshalb schon um 19.00 Uhr zu schlafen, was mir sogleich auch gelang. Doch schon nach einer Stunde war ich wieder wach und krank.

Eine quälende Unruhe plagte mich im ganzen Körper. War es die Sorge um die beschwerliche Tour? War es gar Angst, die mir den Schlaf raubte? Dann hätte ich Arsenicum album einzunehmen. Doch nein, denn ich wusste, dass ich einerseits von einem sehr guten Sherpa geführt wurde und dass ich von Gewohnheit her mich nie scheuen würde, die Tour abzubrechen, sollte sie sich als zu schwierig erweisen. Je länger ich da lag, desto grösser wurde die Unruhe. Ein Verlassen des Schlafsackes kam für mich schon wegen der Kälte, aber auch wegen des steinigen und abfallenden Geländes und der Dunkelheit, nicht in Frage.

Doch schlimmer als die Unruhe war ein Kopfschmerz, wie ich ihn kaum je erlebt hatte und der mir jede Motivation zu Höherem nahm. Meine Empfindungen fokussierten sich zuletzt nur noch auf diesen Schmerz. Litt ich an einer akuten Höhenkrankheit? Ich war doch schon dreimal auf dem Kiliman- jaro und hatte meine letzte Klettertour in Nepal vor 2 Jahren auf den Jala Peak gemacht, ohne dass ich dabei höhenkrank wurde. Ich erforschte meinen Erfahrungsschatz für homöopathische Höhenmedizin und entschloss mich deshalb, Belladonna einzunehmen. Belladonna hatte ich schon mehrmals im Gebirge bei Begleitpersonen verwendet, es hilft bei dem schneidenden oder bohrenden Höhenkopfschmerz mit Benommenheit und Übelkeit, der dann von einer ataktischen Gangunsicherheit gefolgt wird und sich bei jeder Erschütterung, Anstrengung und in der Sonne verschlimmert. Doch die Einnahme einer C200 bewirkte gar nichts, auch nach einer Stunde nicht.

Dann kam mir Glonoin in den Sinn. Ich hatte vor drei Jahren einem Bergsteiger am Thorong Pass in Nepal Glonoin verabreicht, der eindeutig an einem beginnenden Höhen-Hirnödem litt und 1/2 Stunde nach der Einnahme, nach Einsetzten einer massiven Harnflut, wieder völlig beschwerdefrei war. Glonoin bewährt sich ebenfalls bei diesen bohrenden und klopfenden Kopfschmerzen mit Gangunsicherheit, ebenfalls mit Verschlimmerung durch Bewegung und in der Sonne. Glonoin ist ein Folge- und Komplementärmittel zu Belladonna. Inzwischen war es Mitternacht geworden, ich verspürte nicht die geringste Besserung nach Einnahme von Glonoin C200 und ich war nun entschlossen, in 2 Stunden meinem Sherpa mitzuteilen, dass ich die Tour nicht mehr fortsetzen werde. Mein Stolz und daher auch die Tatsache, dass ich weder Dexamethason noch Nifedipin mitführte, verbot es mir, irgendein Syntheticum einzunehmen. Doch nochmals ging ich in mich und versuchte, trotz der beinahe betäubenden Kopfschmerzen meine Gedanken zu sammeln und zu ordnen.

Der Kopfschmerz war (gemäss meinen Tagebuchnotizen) „bohrend wie bei einem Schädel-Hirn-Trauma“, und er war betäubend. Ich realisierte erst jetzt, dass der Ursprung des Schmerzes im Nackenbereich lag. Leichte Nackenschmerzen hatte ich schon vor Beginn der Tour zu Hause verspürt, doch nun liessen sich am Hals seitlich schmerzhafte Muskeln und Myogeloseknoten palpieren. Auch die Unruhe meiner Glieder und der damit verbundene Drang sie zu bewegen, was ich aber bei den engen Zeltverhältnissen gar nicht durfte, um meinen Sherpa nicht zu stören, liessen mich nun an Arnica denken (Abb. 2/3). Warum aber Arnica, war ich doch stets in meinem „Tramp“ bei gleichzeitig streng kontrollierter Atmung aufgestiegen ohne jemals in die Phase der Übersäuerung einzutreten? Eine Überanstrengung lag doch gar nicht vor, oder doch?

Jogger, bzw. Läufer leiden nicht selten an Schmerzen im Nackenbereich. Daran sind beteiligt u.a. der Musculus trapecius sowie die Musculi scaleni ant., med. und posterior, also die Atemhilfsmuskulatur für die Inspiration. Forcierte, tiefe Atmung, die auf einer Höhe von $5000 \mathrm{~m}$ wegen des um $50 \%$ verringerten Sauerstoffgehaltes beim ungeübten Europäer sicher unabdingbar ist, führt zu einer Überlastung dieser Halsmuskeln, deren Schmerzen sich über den ganzen Schädel ausbreiten können. Die Überanstrengung dieser Muskeln, bzw. die forcierte tiefe Atmung waren somit die Causa für den heftigen Kopfschmerz, wie ich ihn zuvor kaum je gekannt hatte.

Eigentlich hatte ich die Hoffnung, die Besteigung des Island Peak fortzu- 


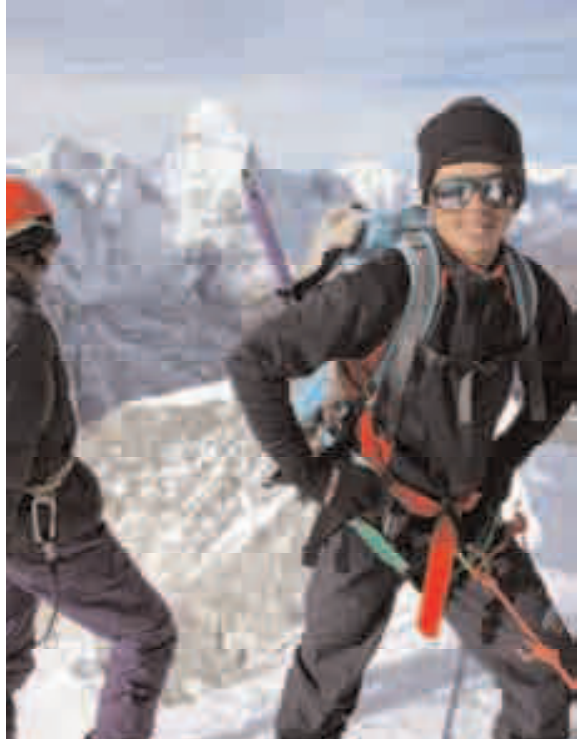

Abb. 2. Dawa Jangbu Sherpa führte, ..

setzen, bereits aufgegeben, wollte aber nochmals einen Versuch mit Arnica C30 Glob. unternehmen. Nun geschah das fast Unglaubliche: Während ich völlig erschlagen dalag, mich im Schlafsack mit dem Schicksal hadernd verkroch und der herannahenden Dinge harrte, spürte ich, deutlich wahrnehmend, wie der Schmerz von Minute $\mathrm{zu}$ Minute nachliess, als hätte ich mir ein Schmerzmittel in die Venen injiziert. Nach etwa $1 / 2$ Stunde war ich beschwerdefrei, verspürte aber, wie der Kopfschmerz langsam wieder einsetzte, aber nun auf der linken Seite; der Schmerz war nicht mehr bohrend, sondern nun eindeutig stechend (Tagebuchnotiz). Sollte ich zuwarten in der Hoffnung, dass Arnica noch wirke, oder sollte ich eine zweite Dosis einnehmen? Ich war überzeugt, dass Arnica ausgewirkt hatte, so dass ich die Dosis repetierte.

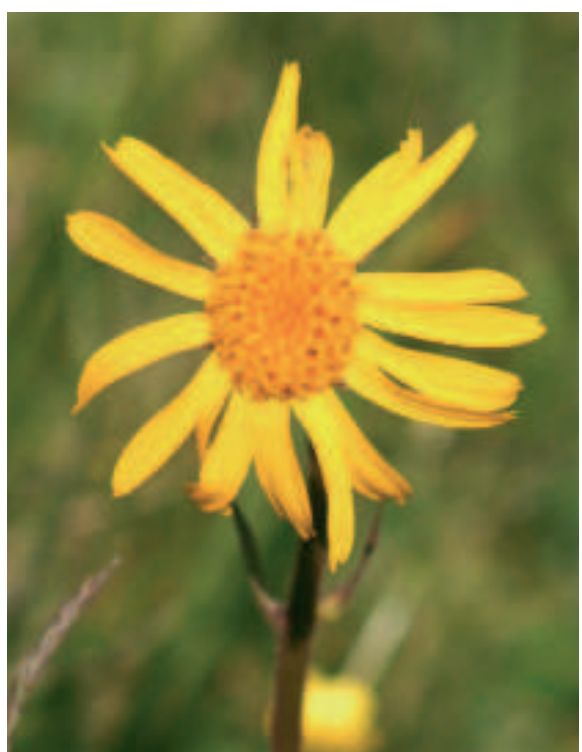

Abb. 3. ... doch Arnica gab die Kraft. (C) R. Saller, Zürich

Und wiederum passierte dasselbe: Innerhalb von Minuten verschwanden alle Schmerzen endgültig und ich fiel gegen $01.00 \mathrm{Uhr}$ in einen tiefen, entspannten Schlaf, bereichert durch angenehme Traumerlebnisse. Nicht einmal die übliche Atemnot, welche sich infolge Reduktion der Atemfrequenz und Atemtiefe beim Eindämmern in Höhenlagen einstellt und so manchen Bergsteiger plagt - welche übrigens sich gut mit Lachesis behandeln lässt stellte sich ein. (Atemnot beim Einschlafen infolge Stockschnupfen mit intensiver Verkrustung der Nasenschleimhaut spricht für Lycopodium). Beschwerdefrei und völlig erholt weckte mich die Weckeruhr um 02.15 Uhr, wie mit meinem Sherpa abgesprochen. Wir kochten uns einen Frühstückstee, ich verzehrte noch einige Kraftriegel, mein Sherpa genoss seine geliebte „garlic soup“, und wir brachen um 03.15 Uhr auf, um den Gipfel zu besteigen, den wir um 07.45 Uhr beschwerdefrei bei herrlichem Wetter kurz nach Sonnenaufgang erreichten.

Dazu gibt es noch eine Nebengeschichte. Von meinem Sherpa weiss ich, dass er nie unter Schlaflosigkeit leidet, dass er selbst auf über 7400 m (Besteigung des Mt. Everest) noch immer im Zelt gut schläft. Vergangene Nacht sei er von Unruhe geplagt gewesen, auch sein buddhistisches Ritual, das er einst im Kloster erlernt hatte, brachte ihm nicht die notwendige Ruhe. Doch plötzlich gegen 01.00 Uhr sei er in einen tiefen, entspannten Schlaf gefallen, bereichert durch angenehme Traumerlebnisse, und schliesslich geweckt durch die Weckeruhr um $02.15 \mathrm{Uhr}$, wie mit mir abgesprochen.

\section{Literatur}

1. Berghold F., Schaffert W.: Handbuch der Trekking- und Expeditionsmedizin. Praxis de Höhenanpassung - Therapie der Höhenkrankheit. DAV Summit Club, München, 3. Auflage 1997

2. Bärtsch $P$, Bailey $D$, Berger $M$, Knauth $M$ Baumgartner R: Acute Mountain Sickess: Controversies and Advances. High Altitude Medicine \& Biology;Vol 5; Num 2; 2004.

3. Hochholzer T: Akute Höhenkrankheit, Höhenlungenöden, Höhenhirnödem.

http://gin.uibk.ac.at/thema/hoehenkrank heit/\#hoehenlungen

4. Boger C.M.: Synoptic Key. Similimum-Verlag Ruppchteroth 2002

5. Farrington EA : Klinische Arzneimittellehre. Ulrich Burgdorf Verlag, Göttingen 1985.

6. Hering C.: Kurzgefasste Arzneimittellehre. Ulrich Burgdorf Verlag, Göttingen 1985.

\section{Korrespondenzadresse:}

Dr. med. Urs Steiner

Allg. Medizin FMH

Staldenstrasse 10, $\mathrm{CH}-6405$ Immensee

dr.urs.steiner@bluewin.ch 